\title{
Optimal Exact Path-Following for Constrained Differentially Flat Systems
}

\author{
Timm Faulwasser* , Veit Hagenmeyer ** and Rolf Findeisen* \\ * Institute for Automation Engineering, \\ Otto-von-Guericke University Magdeburg, Germany \\ ** BASF SE, Ludwigshafen, Germany \\ E-mail: \{timm.faulwasser, rolf.findeisen\}@ovgu.de, \\ veit.hagenmeyer@basf.com
}

\begin{abstract}
We propose a dynamic optimization approach to calculate optimal feedforward controls for exact path-following problems of differentially flat systems. In addition to the derivation of a small dimensional optimal control problem, we provide easily checkable conditions on the existence of inputs guaranteeing that a given path is exactly followable in the presence of constraints on states and inputs. Our approach is based on the projection of the feedforward controlled nonlinear MIMO dynamics along a geometric path onto a linear single-input system in Brunovský normal form. The presented results indicate how the computation of admissible trajectories for set-point changes can be simplified by relying on so called steady state consistent paths. The set-point change of a Van der Vusse reactor is considered as an example.
\end{abstract}

Keywords: Differentially flat systems, input and state constraints, path-following, optimal control.

\section{INTRODUCTION}

Usually, control problems are roughly divided into setpoint stabilization and trajectory tracking problems, whereby the latter means that a time-varying reference is to be tracked. Path-following can be considered as an additional class of problems, in which the reference to be followed is a geometric curve without any preassigned timing information. Path-following problems provide a suitable framework to a plethora of interesting applications: E.g. the control of autonomous vehicles, ships or aircrafts along given geometric references, see Astolfi et al. (2004); Skjetne et al. (2004). Also robot control (Shin and McKay (1985); Verscheure et al. (2009)) as well as batch crystallization (Nagy (2008)) can be formulated as pathfollowing problems. However, the majority of existing results on path-following problems focuses on geometric and Lyapunov-based methods and does not explicitly consider constraints during the controller design. Recent contributions explicitly consider constraints on system inputs and states in a predictive control setup (Faulwasser and Findeisen (2010); Faulwasser et al. (2009)). The aforementioned results share the a priori assumption that a given path is exactly followable.

In the present paper we focus on the class of differentially flat systems (cf. Fliess et al. (1995)) in order to derive both: Easily checkable, sufficient conditions whether a given path is exactly followable in the presence of constraints on states and inputs as well as a small dimensional optimization problem to compute suitable controls. The contributions are as follows: We investigate how the dynamics of a feedforward controlled differentially flat nonlinear MIMO system along a geometric path can be projected to a linear single-input system. Using this projection we show that sufficiently smooth paths in the flat output space of a system are exactly followable in finite time if they can be mapped into the interior of the intersection of the constraints with the set of steady states. Furthermore, we derive a small dimensional dynamic optimization problem to compute the feedforward controls guaranteeing exact path-following. The proposed optimization problem is significantly smaller than existing approaches to exploit flatness in the context of dynamic optimization of constrained systems, cf. Faiz et al. (2001); Oldenburg and Marquardt (2002); Petit et al. (2001); Sira-Ramírez and Agrawal (2004). Related works also exist in the context of computed torque robot control, see Shin and McKay (1985); Verscheure et al. (2009). However, the latter results are limited since robot dynamics are often described by flat systems with a specific structure. Note that we focus on the feedforward control problem. Provided that the model/plant mismatch is sufficiently small, it is straightforward to combine suitable feedforward control signals with a feedback controller in a two-degrees-of-freedom control approach to achieve disturbance attenuation, see e.g. Hagenmeyer and Delaleau (2003); Graichen and Zeitz (2005). After a concise problem statement in Section 2 we present the main contributions in Section 3. In Section 4 we discuss set-point changes of a chemical reactor as an example. Finally, we draw conclusions in Section 5.

\section{Notation}

The image of a set $\mathcal{A} \subset \mathbb{R}^{n}$ under a map $f$ is denoted as $f(\mathcal{A})$. The interior of a compact set $\mathcal{B}$ is written as $\operatorname{int}(\mathcal{B})$. The $k$-th time derivative of a function $r(t)$ is written as $\frac{d^{k} r(t)}{d t^{k}}$ or more conveniently $r^{(k)}$. The $k$-th partial derivative of $g(\tau): \mathbb{R} \mapsto \mathbb{R}^{m}$ with respect to $\tau$ is denoted as $\partial_{\tau}^{k} h \in \mathbb{R}^{m \times 1}$. The set of $k$-times continuously 
differentiable functions is written as $\mathcal{C}^{k}$. The norm $\|x\|$ of $x \in \mathbb{R}^{n}$ denotes the 2-norm. The solution at time $t$ of an ODE $\dot{x}=f(x, u)$ starting at $x(t=0)=x_{0}$ and driven by the input $u$ is denoted as $x\left(t, x_{0} \mid u\right)$. If no ambiguity about the initial condition can arise, we write $x(t \mid u)$.

\section{EXACT FEEDFORWARD PATH-FOLLOWING}

We consider a nonlinear system given by

$$
\begin{aligned}
\dot{x} & =f(x, u), \quad x(0)=x_{0}, \\
y & =h\left(x, u, \dot{u}, \ldots, u^{(l)}\right),
\end{aligned}
$$

where states $x \in \mathbb{R}^{n}$ and inputs $u \in \mathbb{R}^{m}$ are constrained by the compact sets $\mathcal{X} \subset \mathbb{R}^{n}$ and $\mathcal{U} \subset \mathbb{R}^{m}$.

Instead of a set-point or a (time parametrized) reference trajectory we consider a geometric reference to be followed. This reference is denoted as path $\mathcal{P}$ and given as a regular, parametrized curve

$$
\mathcal{P}:=\left\{p \in \mathbb{R}^{m}: \theta \in\left[\theta_{0}, \theta_{T}\right] \subset \mathbb{R} \mapsto p(\theta) \in \mathbb{R}^{m}\right\},
$$

where the parametrization $p(\theta)=\left(p_{1}(\theta), \ldots, p_{m}(\theta)\right)^{T}$ is a priori known. Note that the regularity of a geometric curve implies the bijectivity of the parametrization $\theta \in$ $\mathbb{R} \mapsto p(\theta) \in \mathcal{P}$, cf. Topogonov (2006). Hence regularity excludes cases of self-intersecting paths. In general the path parameter $\theta=\theta(t)$ is time dependent but its time evolution $t \mapsto \theta(t)$ is not given a priori. It has to be determined during the design of the (feedforward) control. This means that the path $\mathcal{P}$ is a geometric reference without any preassigned timing information. However, often one wants to achieve that a system moves forward $(\dot{\theta} \geq 0)$ along $\mathcal{P}$. More concisely we are interested in solving the following control problem.

\section{Problem 1 (Optimal Exact Path-Following)}

Given the constrained system (1), and a path $\mathcal{P}$ of form (2) to be followed. Design an input signal $u(t)$ on $t \in[0, T]$ such that the following conditions are satisfied:

P1 (Exact Path-Following) The system output (1b) moves from a consistent initial condition $\left.h(\cdot)\right|_{t=0}=$ $p\left(\theta_{0}\right)$ in forward direction exactly along the path $\mathcal{P}$. Hence for all $t \in[0, T]$ it holds that $\dot{\theta}(t) \geq 0$ and $h(\cdot) \in \mathcal{P}$.

P2 (Constraint Satisfaction) For all $t \in[0, T]$ the feedforward signal $u(t)$ satisfies the input constraints $u(t) \in \mathcal{U}$, and the corresponding system trajectory satisfies the state constraints $x\left(t, x_{0} \mid u\right) \in \mathcal{X}$.

P3 (Cost Minimization) The feedforward input signal is designed such that the cost functional

$$
J(u, x)=T+\int_{0}^{T} F(x, u) d \tau,
$$

where $F: \mathcal{X} \times \mathcal{U} \mapsto \mathbb{R}, F(\cdot) \in \mathcal{C}^{1}$ is minimized.

For general nonlinear systems the considered problem is challenging for two main reasons: Firstly, minimizing the cost functional (3) subject to the nonlinear MIMO system (1) and the geometric path constraint $\mathcal{P}$ results in a nonlinear optimization problem, e.g. Faulwasser et al. (2009). Secondly, it is in general not easy to verify that an arbitrarily shaped geometric path can be exactly followed if constraints on states and inputs are present. Actually, exact path-following problems are often solved under the a priori assumption that a given $\mathcal{P}$ is exactly followable by the considered system.

In order to circumvent this assumption and tackle the problem we focus on differentially flat systems, cf. Fliess et al. (1995). This enables us to obtain an optimization problem subject to a small dimensional linear SISO system, and to state sufficient conditions for exact pathfollowability in the presence of constraints.

Definition 1 (Differentially Flat System)

Consider the system (1a). If there exists a variable $\xi=$ $\left(\xi_{1}, \ldots, \xi_{m}\right)^{T}$ with $\operatorname{dim} \xi=\operatorname{dim} u$ such that the following statements hold at least locally:

(i) The variable $\xi$ can be written as a function of the state variables $x=\left(x_{1}, \ldots, x_{n}\right)^{T}$, the input variables $u=$ $\left(u_{1}, \ldots, u_{m}\right)^{T}$ and a finite number of time derivatives of the input variables

$$
\xi=g\left(x, u_{1}, \ldots, u_{1}^{\left(l_{1}\right)}, \ldots, u_{m}, \ldots, u_{m}^{\left(l_{m}\right)}\right) .
$$

(ii) The system variables $x$ and $u$ can be expressed as functions of the variable $\xi=\left[\xi_{1}, \ldots, \xi_{m}\right]^{T}$ and a finite number of time-derivatives of $\xi$. Hence

$$
\begin{aligned}
& x=\Phi\left(\xi_{1}, \ldots, \xi_{1}^{\left(k_{1}-1\right)}, \ldots, \xi_{m}, \ldots, \xi^{\left(k_{m}-1\right)}\right), \\
& u=\Psi\left(\xi_{1}, \ldots, \xi_{1}^{\left(k_{1}\right)}, \ldots, \xi_{m}, \ldots, \xi_{m}^{\left(k_{m}\right)}\right) .
\end{aligned}
$$

(iii) The components of $\xi$ are differentially independent, they do not fulfill any differential equation.

Then $\xi$ is called a flat output of (1a). And (1a) is called a (differentially) flat system.

In the following we assume that $y=h(\cdot)$ from $(1 \mathrm{~b})$ is a flat output of (1). We denote the evaluation of (4b) or (4c) along a specific sufficiently smooth output trajectory $y(t)$ as $x=\left.\Phi(\cdot)\right|_{y(t)}$ and $u=\left.\Psi(\cdot)\right|_{y(t)}$ respectively.

It is well-known that trajectory tracking or set-point changes can be achieved easily for flat systems, cf. Fliess et al. (1995); Lévine (2009); Sira-Ramírez and Agrawal (2004). If a sufficiently smooth reference trajectory is known a priori, one basically exploits (4c) and obtains a suitable feedforward control. Consequently, flatness can also be used to state sufficient conditions for unconstrained exact path-following of flat systems.

Lemma 1

Given an unconstrained nonlinear flat system (1a) and any regular path $\mathcal{P}$ specified by an a priori known parametrization $p(\theta)$ in a flat output space (1b).

\section{Suppose that}

(i) the parametrization $p(\theta) \in \mathcal{C}^{\hat{k}}$, where $\hat{k}=\max \left\{k_{i}\right\}$ and the constants $k_{i} \in \mathbb{N}$ are defined by (4c);

(ii) the initial condition $x_{0}$ of (1a) is consistent with $\mathcal{P}$ and $\theta(t) \in \mathcal{C}^{\hat{k}}$, such that

$$
x_{0}=\left.\Phi(\cdot)\right|_{p(\theta(t=0))},
$$

where $\dot{\theta}(t) \geq 0$ and $\theta(0)=\theta_{0}$ and $\theta(T)=\theta_{T}$.

Then the feedforward input

$$
u=\Psi\left(p_{1}(\theta(t)), \ldots, \frac{d^{k_{1}} p_{1}}{d t^{k_{1}}}, \ldots, p_{m}(\theta(t)), \ldots, \frac{d^{k_{m}} p_{m}}{d t^{k_{m}}}\right)
$$

guarantees that the system $\dot{x}=f(x, u)$, starting from $x_{0}$, follows the path $\mathcal{P}$ exactly in forward direction. 
The proof of this lemma follows directly by calculation of the time derivatives of $p(\theta(t))$, use of $(4 \mathrm{c})$ and the ideas presented in Hagenmeyer and Delaleau (2003).

\section{MAIN RESULTS}

The challenging part is to extend Lemma 1 such that constraints on inputs and states can be considered. We start with a technical lemma. It is subsequently used to project the feedforward controlled nonlinear MIMO dynamics (1) along the path $\mathcal{P}$ to a linear single-input system in Brunovský normal form. Finally, the projection is used to obtain a small dimensional optimization problem and sufficient conditions for exact path-followability.

\section{Lemma 2}

Given a regular path $\mathcal{P} \subset \mathbb{R}^{m}$ from (2) and its $k$-times continuously differentiable parametrization $\theta \mapsto p(\theta)$. Suppose that the time evolution $t \mapsto \theta(t)$ is also $k$-times continuously differentiable.

Then the map

$$
\Delta:\left(\theta, \dot{\theta}, \ldots, \theta^{(k)}\right) \in \mathbb{R}^{k} \mapsto\left(p, \dot{p}, \ldots, p^{(k)}\right) \in \mathbb{R}^{m \times k}
$$

given by $p(\theta(t))$ and its time derivatives is invertible for all values of $\left(p, \dot{p}, \ldots, p^{k}\right)^{T}$ which are contained in the image of $\Delta$.

Proof. The regularity of $\mathcal{P}$ implies the bijectivity of $\theta \mapsto p(\theta)$ and $\operatorname{rank}\left(\partial_{\theta} p(\theta)\right)=1$ for all $\theta$, cf. Topogonov (2006). Therefore, given the parametrization $p(\theta)$ and a specific point $p \in \mathcal{P}$, the equation $p=p(\theta)$ can be solved for the unique value of $\theta$. Due to the rank condition it follows that at least one component of $\partial_{\theta} p(\theta)=$ $\left(\partial_{\theta} p_{1}(\theta), \ldots, \partial_{\theta} p_{i}(\theta), \ldots, \partial_{\theta} p_{m}(\theta)\right)^{T}$ is not equal to zero. Hence one can solve $\dot{p}_{i}=\partial_{\theta} p_{i}(\theta) \cdot \dot{\theta}$ for $\dot{\theta}$. Using the equation for $\ddot{p}(\theta)$ and the previously calculated values of $\theta$ and $\dot{\theta}$, one can determine $\ddot{\theta}$ from $\ddot{p}_{i}-\partial_{\theta}^{2} p_{i}(\theta) \cdot \dot{\theta}^{2}=\partial_{\theta} p_{i}(\theta) \cdot \ddot{\theta}$. Following this procedure for the remaining time derivatives of $p(\theta)$ one obtains the unique values of $\ddot{\theta}, \ldots, \theta^{(k)}$.

The following theorem sets the conceptual basis for the design approach considered in this note.

Theorem 1 (Equivalence to SISO System)

Given a nonlinear flat system (1) and a regular path $\mathcal{P}$ specified by an a priori known parametrization $p(\theta)$ in a flat output space of the system. Suppose that conditions (i) and (ii) of Lemma 1 are satisfied.

Then the dynamics of the nonlinear MIMO system (1) under the feedforward control $u=\left.\Psi(\cdot)\right|_{p(\theta(t))}$ given by (6), where $\theta(t)$ is of class $\mathcal{C}^{\hat{k}}$, are equivalent to a linear, single input system in Brunovský normal form.

Proof. Provided that condition (i) of Lemma 1 is satisfied, any choice of a class $\mathcal{C}^{\hat{k}}$ timing $\theta(t)$ specifies a sufficiently often continuously differentiable output reference trajectory for the flat system (1). It follows that system (1) under the feedforward control (6) is equivalent to a linear MIMO system in Brunovský normal form such that for all $i=1, \ldots, m$

$$
\begin{array}{ccc}
\dot{\xi}_{i, 1} & = & \xi_{i, 2} \\
\vdots & \vdots \\
\dot{\xi}_{i, k_{i}} & = & \frac{d^{k_{i}} p_{i}(\theta(t))}{d t^{k_{i}}}
\end{array}
$$

where the time derivatives $\frac{d^{k_{i}} p_{i}(\theta(t))}{d t^{k_{i}}}$ are the inputs, see Hagenmeyer and Delaleau (2003).

On the one hand the input and state parametrizations (4bc) reveal that any choice of $\theta(t) \in \mathcal{C}^{\hat{k}}$ leads to a unique state evolution of (1). On the other hand Lemma 2 states that from the knowledge of the parametrization $p(\theta)$ and the values $p, \dot{p}, \ldots, p^{(\hat{k})}$ the values of $\theta, \dot{\theta}, \ldots, \theta^{(\hat{k})}$ can be uniquely determined. Hence the dynamics of the system (1) along a given regular path $\mathcal{P}$ are uniquely described by the choice of a class $\mathcal{C}^{\hat{k}}$ timing $\theta(t)$. Therefore the MIMO Brunovský normal form reduces to the following SISO Brunovský normal form

$$
\begin{array}{ccc}
\dot{z}_{1} & = & z_{2} \\
\dot{z}_{2} & = & z_{3} \\
\vdots & & \vdots \\
\dot{z}_{\hat{k}+1}= & \theta^{(\hat{k}+1)}(t),
\end{array}
$$

where the $\hat{k}+1$-th time derivative of $\theta(t)$ can be regarded as free input variable.

Since system (1) under the feedforward control $u=$ $\left.\Psi(\cdot)\right|_{p(\theta(t))}$ is equivalent to (7) and the latter is equivalent to (8) it follows that (1) is also equivalent to (8).

\section{Proposed Optimal Control Problem}

The previous considerations show that the feedforward input $u=\left.\Psi(\cdot)\right|_{p(\theta(t))}$ can be reformulated as a function of $\theta(t)$ and its first $\hat{k}$ time derivatives. Equivalently one may use the representation via the SISO system (8). Based on the substitution

$$
z:=\left(z_{1}, z_{2}, \ldots, z_{\hat{k}+1}\right)^{T}=\left(\theta, \dot{\theta}, \ldots, \theta^{(\hat{k})}\right)^{T}
$$

one can represent the feedforward input $u$ from (6) and the state parametrization $(4 \mathrm{~b})$ as

$$
\begin{array}{ll}
u=\Psi\left(\theta, \dot{\theta}, \ldots, \theta^{(\hat{k})}\right) & =: \Psi(z), \\
x=\Phi\left(\theta, \dot{\theta}, \ldots, \theta^{(\hat{k}-1)}\right) & =: \Phi(z) .
\end{array}
$$

Next, we use the $\hat{k}+1$-th time derivative of $\theta(t)$ as decision variable in a suitably chosen optimal control problem. To simplify the subsequent notation we set

$$
A:=\left(\begin{array}{c|c}
0 & I^{\hat{k} \times \hat{k}} \\
\hline 0 & 0
\end{array}\right), \quad B:=(0, \ldots, 0,1)^{T},
$$

where $A \in \mathbb{R}^{(\hat{k}+1) \times(\hat{k}+1)}$ and $B \in \mathbb{R}^{\hat{k}+1}$. Consider the feedforward path-following task (P1-P3) for (1a). Taking the reformulation into account an optimal feedforward input signal, which respects the constraints $x \in \mathcal{X}, u \in \mathcal{U}$, is given by the solution of the following optimal control problem:

$$
\min _{v, T} T+\int_{0}^{T} F(x, u) d \tau
$$

subject to the dynamics

$$
\dot{z}=A z+B v,
$$

the (convex) state constraints

$$
\begin{aligned}
z(0) & =\left[\theta_{0}, 0, \ldots, 0\right]^{T}, \\
z(T) & =\left[\theta_{T}, 0, \ldots, 0\right]^{T}, \\
\forall t \in[0, T]: \quad z_{2} & \geq 0,
\end{aligned}
$$


and the additional constraints

$$
\begin{array}{ll}
\forall t \in[0, T]: & u=\Psi(z(t)) \in \mathcal{U}, \\
\forall t \in[0, T]: & x=\Phi(z(t)) \in \mathcal{X}, \\
\forall t \in[0, T]: & v \in \mathcal{V} \subset \mathbb{R} .
\end{array}
$$

Note that the proposed optimal control problem has a small number of linearly coupled dynamic states. Scalar input $v(t)$ and free end time $T$ are decision variables.

The cost functional (11a) directly expresses the requirement from P3. The constraints (11c) and (11d) state that the initial path point is $p\left(\theta_{0}\right)$ and the final path point is $p\left(\theta_{T}\right)$. Equation (11e) expresses the condition on forward movement along the path (P1). The constraints on states and inputs of the flat system (1a) $u=\Psi(z) \in$ $\mathcal{U}, x=\Phi(z) \in \mathcal{X}$ are considered via $(11 \mathrm{f}, \mathrm{g})$, cf. part P2 of the problem statement. In order to achieve that the original system (1) follows the path $\mathcal{P}$ exactly, it suffices to compute any admissible solution $v$ to (11) and the corresponding evolution of $z(t \mid v)$. Note that the optimal solution $v^{\star}(t)$ is the input to the virtual system (11b). Finally, the input applied to the nonlinear differentially flat system (1a) is calculated from the optimal evolution of $z\left(t \mid v^{\star}\right)$ via the input parametrization $u=\Psi(z)$ and the knowledge about the path parametrization $p(\theta)$ from $(6)$.

For general flat systems and arbitrary paths $\mathcal{P}$ the constraints of the proposed optimal control problem will usually be non-convex due to the nonlinear maps $\Psi(\cdot)$ and $\Phi(\cdot)$ from (11f,g). Nevertheless, under fairly mild assumptions the existence of optimal solutions to (11) in the presence of constraints on states and inputs of the original system (1) can be guaranteed. In order to do this, we investigate the relation between a path $\mathcal{P}$, the set of steady states and the constraints of (1).

Definition 2 (Steady State Consistent Path)

We call a path $\mathcal{P}$ from (2) weakly steady state consistent with respect to system (1) and its constraints $\mathcal{X}$ and $\mathcal{U}$, if for all $\theta \in\left[\theta_{0}, \theta_{T}\right]$ exist $x_{s} \in \mathcal{X}$ and constant $u_{s} \in \mathcal{U}$ s. t.

$$
\begin{aligned}
0 & =f\left(x_{s}, u_{s}\right), \\
p(\theta) & =h\left(x_{s}, u_{s}, 0, \ldots, 0\right)
\end{aligned}
$$

hold.

If additionally for all $\theta \in\left[\theta_{0}, \theta_{T}\right]$ it can be verified that $x_{s} \in \operatorname{int}(\mathcal{X})$ and $u_{s} \in \operatorname{int}(\mathcal{U})$ then we call $\mathcal{P}$ strongly steady state consistent.

Let $\mathcal{Z} \subset \mathbb{R}^{\hat{k}+1}$ be the set of all states $z$ which satisfy the constraints (11c-e) then the following theorem holds.

Theorem 2 (Exact Path-Followability)

Given a flat system (1), a path $\mathcal{P}(2)$ and the corresponding optimal control problem (11). Suppose that conditions (i) and (ii) of Lemma 1 are satisfied. And for all $z \in \mathcal{Z} \subset \mathbb{R}^{\hat{k}+1}$

(i) the maps $\Psi: z \in \mathbb{R}^{\hat{k}+1} \mapsto u \in \mathbb{R}^{m}$ and $\Phi: z \in$ $\mathbb{R}^{\hat{k}+1} \mapsto x \in \mathbb{R}^{n}$ from (10) are continuous;

(ii) and the path $\mathcal{P}$ is strongly steady state consistent to (1) and the constraint sets $\mathcal{X}, \mathcal{U}$.

Then $\mathcal{P}$ is exactly followable by system (1) s.t. the constraints $x \in \mathcal{X}$ and $u \in \mathcal{U}$ are satisfied and the corresponding problem (11) has an optimal solution. Furthermore, if the minimum-time case $(F(\cdot)=0)$ is considered, then the minimal transition time $T^{\star}$ is finite.
Proof. Our proof is based on the construction of an admissible solution to (11) and proceeds in four steps. Firstly, we express the supposition of strongly path consistency in terms of the flat state and input parametrizations and perform a suitable reformulation. Secondly, we split the integrator chain (11b) into two parts and consider the last integrator as a flat system. This leads to the design of an admissible reference signal for $z_{2}(t)=\dot{\theta}$ which guarantees that $\theta_{T}$ is reached in finite time. In the third step, we consider the remaining $\hat{k}$ integrators again as a flat system and design an admissible $v$ such that the desired $z_{2}(t)$ trajectory is perfectly tracked. Finally, we conclude from the existence of an admissible input $v$ to the existence of an optimal finite-time solution to (11).

Step 1: In terms of the flat input and state parametrization a strongly steady state consistent path means that for all $z_{1} \in\left[\theta_{0}, \theta_{T}\right]$

$$
\begin{aligned}
& \Phi\left(z_{1}, 0, \ldots, 0\right) \in \operatorname{int}(\mathcal{X}) \\
& \Psi\left(z_{1}, 0, \ldots, 0\right) \in \operatorname{int}(\mathcal{U})
\end{aligned}
$$

In the following we focus solely on the constraint $\Psi(\cdot) \in \mathcal{U}$ since the consideration of $\Phi(\cdot) \in \mathcal{X}$ relies on the same concept. Consider the set $\tilde{\mathcal{Z}}:=\left[\theta_{0}, \theta_{T}\right] \times\left[0, c_{2}\right] \times\left[-c_{3}, c_{3}\right] \times$ $\cdots \times\left[-c_{\hat{k}+1}, c_{\hat{k}+1}\right]$, where $\sum_{i=2}^{\hat{k}+1} c_{i}^{2} \leq \delta$. Due to the continuity of $\Psi(\cdot)$ there exists a sufficiently small $\delta>0$ such that the image set of $\tilde{\mathcal{Z}}$ under $\Psi$ lies completely in the interior of $\mathcal{U}$. Hence $\Psi(\tilde{\mathcal{Z}}) \in \operatorname{int}(\mathcal{U})$.

The main idea is to rely on the tightened constraint set $\tilde{\mathcal{Z}}$ since keeping $z(t)$ in $\tilde{\mathcal{Z}}$ suffices to satisfy (11f). Choose the constants $c_{i}$ with $i=2, \ldots, \hat{k}+1$ such that all $c_{i}>0$ and $\sum_{i=2}^{\hat{k}+1} c_{i}^{2} \leq \delta$. W.l.o.g. assume that there exists a function $\gamma(t) \in \mathbb{R}$, defined on $t \in[0, s], 0<s<\frac{\theta_{T}-\theta_{0}}{2 c_{2}}$, which is monotonously increasing $\dot{\gamma}(t) \geq 0$ and $\gamma(t) \in \mathcal{C}^{\hat{k}}$. Furthermore, this function can be chosen such that $\gamma(0)=$ $0, \gamma(s)=c_{2},\left.\gamma^{(i)}(t)\right|_{0}=\left.\gamma^{(i)}(t)\right|_{s}=0$ and $\gamma^{(i)} \in\left[-c_{i}, c_{i}\right]$ for all $i=3, \ldots, \hat{k}+1$ hold. Basically, $\gamma(t)$ is a sufficiently smooth signal which increases from 0 to $c_{2}$ during some finite time $s$, while its time derivatives remain bounded. Using $\gamma(t)$ we can construct a reference motion for $z_{2}(t)$.

Step 2: Consider the last part of the integrator chain (11b) and denote $z_{2}$ as $w$, hence $\dot{z}_{1}=w$. Next, we design a signal $w \in\left[0, c_{2}\right]$ which is sufficiently often continuously differentiable $\left(w \in \mathcal{C}^{\hat{k}}\right)$ and ensures for some $T<\infty$ that $z_{1}\left(T, \theta_{0} \mid w\right)=\theta_{T}$. Using the function $\gamma(t)$ introduced before we choose $w$ as

$$
w(t, T)= \begin{cases}\gamma(t), & t \in[0, s] \\ c_{2}, & t \in(s, s+T) \\ c_{2}-\gamma(t), & t \in[s+T, 2 s+T]\end{cases}
$$

Due to the properties of $\gamma(t)$ the signal $w(t, T)$ is in $\mathcal{C}^{\hat{k}}$, $w(t, T) \geq 0$ for all $t \in[0,2 s+T]$ and $\left.w^{(i)}(t, T)\right|_{0}=$ $\left.w^{(i)}(t, T)\right|_{2 s+T}=0$ for $i=1, \ldots, \hat{k}$. For any $\infty>T \geq$ $\frac{\theta_{T}-\theta_{0}}{c_{2}}$ it follows

$$
z_{1}(2 s+T \mid w(t, T))=\theta_{0}+\int_{0}^{2 s+T} w(\tau, T) d \tau>\theta_{T} .
$$

The inequality follows directly from the construction of $w(t, T)$. Furthermore, $s$ and $T$ are finite and so is $2 s+T$. Therefore we know from the mean value theorem that 
for some finite $T^{\circ}: 0 \leq T^{\circ}<T$ the value $z_{1}(2 s+$ $\left.T^{\circ} \mid w\left(t, T^{\circ}\right)\right)=\theta_{T}$. It is clear that $w\left(t, T^{\circ}\right) \in \mathcal{C}^{\hat{k}}$ and $w\left(t, T^{\circ}\right) \geq 0$.

Step 3: Note that the complete integrator chain (11b) is linear and controllable and hence differentially flat, cf. Fliess et al. (1995). A flat output of (11b) is given by $z_{1}$. Indeed also the $\hat{k}$ last parts of the integrator chain $z_{2}^{(\hat{k})}(t)=v$ can be regarded as a flat system, where $z_{2}$ is the considered flat output. In order to design the desired input we set $v^{\circ}=w^{(\hat{k})}\left(t, T^{\circ}\right)$. Since $w\left(t, T^{\circ}\right) \in \mathcal{C}^{\hat{k}}$ it follows that $w^{(\hat{k})}\left(t, T^{\circ}\right) \in \mathcal{V}$, where $\mathcal{V}$ is some closed interval of $\mathbb{R}$.

Step 4: The existence of an optimal solution to (11) can be deduced from two properties: The existence of at least one admissible solution to the problem and the convexity and compactness of the extended velocity set $\mathcal{S}:=\{v \in \mathcal{V} \mapsto$ $\left(A z+B v, F(\Phi(z), \Psi(z)) \in \mathbb{R}^{\hat{k}+2}\right\}$ for all fixed $z$. Linearity of (11b) and compactness of $\mathcal{V} \subset \mathbb{R}$ imply compactness and convexity of $\mathcal{S}$.

In the preceding steps we have constructed an admissible input $v^{\circ}$ for (11) which guarantees that the solution $z\left(t \mid v^{\circ}\right)$ satisfies the tightened constraints $\tilde{\mathcal{Z}}$, therefore $u=$ $\Psi\left(z\left(t \mid v^{\circ}\right)\right) \in \operatorname{int}(\mathcal{U})$ satisfies the constraint (11f). Hence we can conclude from the existence of an admissible solution to the existence of an optimal solution to problem (11).

Furthermore, we can deduce that if the minimum time case $(F(\cdot)=0)$ is considered, then $v^{\circ}$ guarantees that for some finite $T^{\circ}: z_{1}\left(2 s+T^{\circ} \mid v^{\circ}\right)=\theta_{T}$. It follows $0 \leq T^{\star}<2 s+T^{\circ}<\infty$. Hence the minimal transition time $T^{\star}$ is finite. This finishes the proof.

Remark 1

Note that strong steady state consistency of a path $\mathcal{P}$ in the sense of Definition 2 combined with the continuity of input and state parametrizations of a flat system is merely a sufficient condition. However, if $\mathcal{P}$ is only weakly steady state consistent, then it is in general difficult to guarantee constraint satisfaction. In that case even a slow motion along the path might cause violation of the constraints.

The proposed optimization approach can be understood as a projection of the feedforward controlled MIMO dynamics onto the 1 -dimensional path $\mathcal{P}$. Main advantages of this projection are that the optimization problem (11) is subject to quite small dimensional linear dynamics. Furthermore, the projection enables us to state sufficient conditions for exact path-followability as conditions guaranteeing the existence of admissible solutions to (11).

\section{PATH-FOLLOWING FOR A CHEMICAL REACTOR}

As an example we consider a Van der Vusse CSTR reactor described by the dynamics (Rothfuss et al. (1996))

$$
\begin{aligned}
\dot{c}_{A} & =r_{A}\left(c_{A}, T\right)+\left(c_{I n}-c_{A}\right) u_{1} \\
\dot{c}_{B} & =r_{B}\left(c_{A}, c_{B}, T\right)-c_{B} u_{1} \\
\dot{T} & =h\left(c_{A}, c_{B}, T\right)+\alpha\left(u_{2}-T\right)+\left(T_{I n}-T\right) u_{1},
\end{aligned}
$$

where

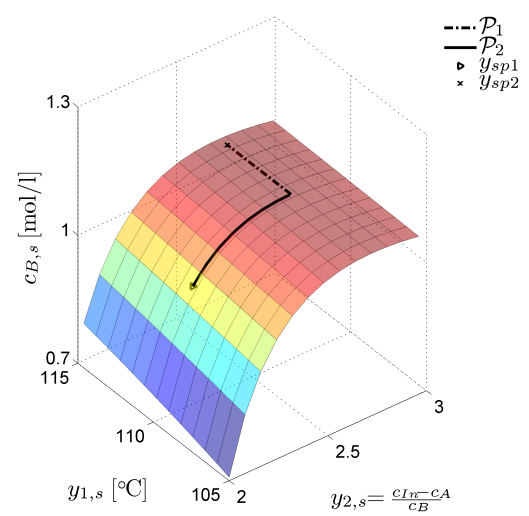

Fig. 1. Path in surface of stationary product concentration. a) state evolution
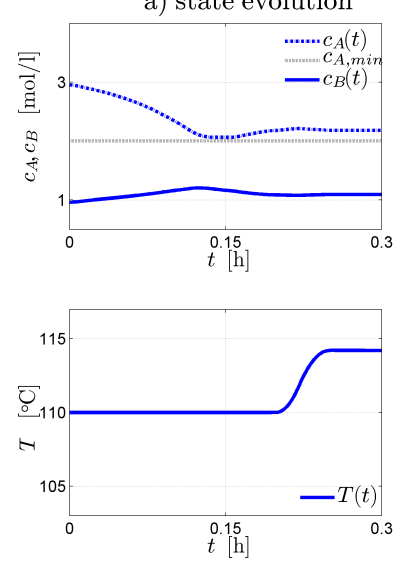

b) system inputs
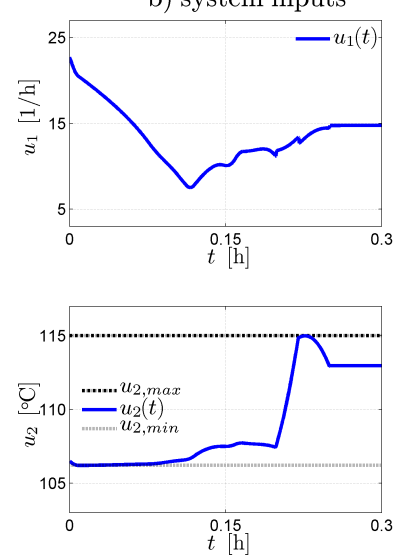

Fig. 2. a) state evolution, b) optimal inputs.

$$
\begin{aligned}
r_{A}(\cdot) & =-k_{1}(T) c_{A}-k_{2}(T) c_{A}^{2} \\
r_{A}(\cdot) & =k_{1}(T)\left(c_{A}-c_{B}\right) \\
h(\cdot) & =-\delta\left(k_{1}(T)\left(c_{A} H_{A B}+c_{B} H_{B C}\right)+k_{2}(T) c_{A}^{2} H_{A D}\right)
\end{aligned}
$$

and the reactions kinetics are of Arrhenius type $k_{i}(T)=$ $k_{i 0} e^{\frac{-E_{i}}{T+T_{0}}}, \quad i=1,2$. The system states $c_{A}$ and $c_{B}$ refer to the educt and product concentration in the CSTR, $T$ is the reactor temperature. The educt concentration is subject to the constraint $2[\mathrm{~mol} / \mathrm{l}] \leq c_{A} \leq 10[\mathrm{~mol} / \mathrm{l}]$. The input $u_{1} \geq 0[1 / \mathrm{h}]$ is the normalized flow rate through the reactor and $u_{2} \in\left[106.2^{\circ} \mathrm{C}, 115^{\circ} \mathrm{C}\right]$ refers to the temperature in the cooling jacket. It can be shown that

$$
y=\left(T, \frac{c_{I n}-c_{A}}{c_{B}}\right)^{T}
$$

is a flat output of (14). Since the calculation of the flat state and input parametrizations $(4 \mathrm{~b}, \mathrm{c})$ leads to vast and complex expressions, we give here only functional dependencies. Considering the flat output (15) the inputs can be expressed as

$$
\begin{aligned}
& u_{1}=\Psi_{1}\left(y_{1}, y_{2}, \dot{y}_{1}, \dot{y}_{2}, \ddot{y}_{2}\right), \\
& u_{2}=\Psi_{2}\left(y_{1}, y_{2}, \dot{y}_{1}, \dot{y}_{2}, \ddot{y}_{2}\right) .
\end{aligned}
$$

The considered path-following problem was proposed in Rothfuss et al. (1996), where a more detailed presentation and the system parameters can be found. The task is to perform a fast set-point change in the surface of stationary product concentration $c_{B, s}$ in two steps as depicted in 
Figure 1. The first step leads from the set-point $y_{S P 1}=$ $\left(110^{\circ} \mathrm{C}, 2.2\right)^{T}$ along the path $\mathcal{P}_{1}$ defined by the affine parametrization

$$
\mathcal{P}_{1}: \theta \in[2.2,2.69] \mapsto p(\theta)=\left(110^{\circ} \mathrm{C}, \theta\right)^{T} .
$$

The second step is to move along the path of maximal steady state product concentration given by the nonlinear parametrization

$$
\mathcal{P}_{2}: \theta \in\left[110^{\circ} \mathrm{C}, 114.21^{\circ} \mathrm{C}\right] \mapsto p(\theta)=\left(2 \sqrt{\frac{k_{1}(\theta)+c_{I n} k_{2}(\theta)}{k_{1}(\theta)}}\right)
$$

to the final set-point $y_{S P 2}=\left(114.21^{\circ} \mathrm{C}, 2.69\right)^{T}$.

Relying on the input parametrization (16) and the path descriptions for $\mathcal{P}_{1,2}$, it is easy to check numerically that the considered path is strongly steady state consistent with respect to the considered input and state constraints. Since the flat input parametrization from (16) depends on $\ddot{y}_{2}$ as highest output derivative the virtual dynamics (11b) are an integrator chain of length three. The set-point change should be achieved reasonably fast, therefore the cost $F(\cdot)=0$ is used in (11a).

In order to compute feedforward controls which nominally move the system (14) from one set-point to the other we apply the proposed approach for both paths. The simulations are carried out with Matlab and a multipleshooting implementation available in the ACADO optimization toolbox, see Ariens et al. (2010). Solving (11) for both paths $\mathcal{P}_{1,2}$ separately leads to the solutions depicted in Figure 2. Part a) shows the state evolutions of the original system (14), part b) presents the corresponding input signals. $\mathcal{P}_{1}$ is accomplished in less then $0.2 \mathrm{~h}$ while steering the system along $\mathcal{P}_{2}$ needs about $0.06 \mathrm{~h}$. The simulation results show that the obtained solution respects the considered constraints. Additionally, the overall transition time $\approx 0.26 \mathrm{~h}$ is much shorter than the heuristic solution of 1.0h presented in Rothfuss et al. (1996).

\section{CONCLUSIONS}

In this contribution we investigate path-following problems for constrained differentially flat systems. We show that a feedforward controlled differentially flat nonlinear MIMO system can be projected onto a linear single-input system. Based on this projection we propose a small dimensional optimization problem, which can be efficiently solved in order to compute optimal feedforward control signals for exact path-following. Additionally, we derive sufficient conditions for the problem of steering a constrained flat system exactly along a given geometric path in a flat output space. The considered approach shows how the calculation of suitable feedforward inputs for setpoint changes can be simplified by relying on steady state consistent paths.

\section{ACKNOWLEDGEMENTS}

The authors gratefully acknowledge the helpful comments of Friedrich von Haeseler, Philipp Rumschinski and Benjamin Kern.

\section{REFERENCES}

Ariens, D., Houska, B., Ferreau, H., and Logist, F. (2010). $A C A D O$ for Matlab User's Manual. Optimization in
Engineering Center (OPTEC), 1.0beta edition. URL http: //www . acadotoolkit.org/.

Astolfi, A., Bolzern, P., and Locatelli, A. (2004). Pathtracking of a tractor-trailer vehicle along rectilinear and circular paths: a lyapunov-based approach. IEEE Trans. Robot. and Autom., 20(1), 154-160.

Faiz, N., Agrawal, S., and Murray, R. (2001). Trajectory planning of differentially flat systems with dynamics and inequalities. J. Guid. Contr. Dyn., 24(2), 219-227.

Faulwasser, T. and Findeisen, R. (2010). Constrained output path-following for nonlinear systems using predictive control. In Proc. of 8th IFAC Symposium on Nonlinear Control Systems (NOLCOS), Bologna, Italy, 753-758.

Faulwasser, T., Kern, B., and Findeisen, R. (2009). Model predictive path-following for constrained nonlinear systems. In Proc. 48th IEEE Conf. on Decision and Control held jointly with the 2009 28th Chinese Control Conf. $C D C / C C C$ 2009, 8642-8647.

Fliess, M., Lévine, J., Martin, P., and Rouchon, P. (1995). Flatness and defect of non-linear systems: introductory theory and examples. Int. J. Contr., 61(6), 1327-1361.

Graichen, K. and Zeitz, M. (2005). Feedforward control design for nonlinear control systems under input constraints. In T. Meurer, K. Graichen, and E. Gilles (eds.), Control and Observer Design for Nonlinear Finite and Infinite Dimensional Systems, Lecture Notes in Control and Information Sciences 322, 235-252. Springer, Berlin.

Hagenmeyer, V. and Delaleau, E. (2003). Exact feedforward linearization based on differential flatness. Int. J. Cont., 76(6), 537-556.

Lévine, J. (2009). Analysis and control of nonlinear systems: a flatness-based approach. Mathematical Engineering. Springer, Berlin.

Nagy, Z. (2008). Model based control approach for batch crystallization product design. In Proc. 17th IFAC World Congress. Seoul, Korea.

Oldenburg, J. and Marquardt, W. (2002). Flatness and higher order differential model representations in dynamic optimization. Comp. \& Chem. Eng., 26(3), 385 -400 .

Petit, N., Milam, M., and Murray, R. (2001). Inversion based constrained trajectory optimization. In Proc. of 5th IFAC symposium on nonlinear control systems (NOLCOS).

Rothfuss, R., Rudolph, J., and Zeitz, M. (1996). Flatness based control of a nonlinear chemical reactor model. Automatica, 32, 1433-1439.

Shin, K. and McKay, N. (1985). Minimum-time control of robotic manipulators with geometric path constraints. IEEE Trans. Automat. Contr., 30(6), 531 - 541.

Sira-Ramírez, H. and Agrawal, S. (2004). Differentially flat systems. Control Engineering Series. Marcel Dekker Inc, New York.

Skjetne, R., Fossen, T., and Kokotovic, P. (2004). Robust output maneuvering for a class of nonlinear systems. Automatica, 40(3), 373-383.

Topogonov, V. (2006). Differential Geometry of Curves and Surfaces - A Concise Guide. Birkhäuser, Boston.

Verscheure, D., Demeulenaere, B., Swevers, J., De Schutter, J., and Diehl, M. (2009). Time-optimal path tracking for robots: A convex optimization approach. IEEE Trans. Automat. Contr., 54(10), 2318-2327. 